\title{
Is it possible to predict gas yields of any biomass after rapid pyrolysis at high temperature from its composition in cellulose, hemicellulose and lignin?
}

\author{
Carole Couhert*, Jean-Michel Commandre, Sylvain Salvador \\ Université de Toulouse, Ecole des Mines d'Albi - Carmaux, RAPSODEE UMR CNRS 2392, Campus Jarlard, Albi F 81013, France
}

\begin{abstract}
A B S T R A C T
Ligno-cellulosic biomass from different sources presents very variable compositions. Consequently, there is a wide variation in the nature and quantities of gaseous products obtained after thermal treatment of biomasses.

The objective of this work is to establish a link between the composition of a biomass and its pyrolysis gas yields and composition. Experimental flash pyrolysis of several biomasses at a temperature of $950{ }^{\circ} \mathrm{C}$ and a gas residence time of about $2 \mathrm{~s}$ was carried out. An attempt was then made to predict gas yields of any biomass according to its composition. We show that an additivity law does not allow the gas yields of a biomass to be correlated with its fractions of cellulose, hemicellulose and lignin. Several potential explanations are then offered and quantitatively demonstrated: it is shown that interactions occur between compounds and that mineral matter influences the pyrolysis process.
\end{abstract}

Keywords:

Flash pyrolysis

Biomass

Components

Ash

\section{Introduction}

Biomass is today considered as a renewable fuel, whose use would help to reduce greenhouse gases. Over the past two decades, worldwide interest has been growing in the thermochemical conversion of renewable resources through pyrolysis or gasification [1].

There has been a great interest in pyrolysis, especially fast pyrolysis of biomass to maximise gas production. Pyrolysis can be a process in itself, and is also the first step of any gasification process. Several studies in this field have looked at the effect of experimental parameters on product distribution. The three principal parameters studied are temperature, heating rate and the nature of the biomass.

When the temperature of pyrolysis increases, the yields of liquids and char decrease and the gas yields increase. Cracking of liquid hydrocarbons favours the formation of gases, and cracking of gaseous hydrocarbons favours the formation of $\mathrm{H}_{2}$ [2-6]. The higher the pyrolysis temperature, the higher the $\mathrm{CO}$ and $\mathrm{H}_{2}$ yields and the lower the $\mathrm{CH}_{4}$ and $\mathrm{CO}_{2}$ yields [3,4].

When the heating rate increases, at low temperature (up to $500{ }^{\circ} \mathrm{C}$ ), gas and char yields decrease whereas liquid yield increases; at high temperature, char and liquids yields decrease while gas yield increases [5,7].

But the nature of the biomass can also influence the quantities and composition of products. Biomass is made up of many differ-

\footnotetext{
* Corresponding author

E-mail address: couhert@enstimac.fr (C. Couhert).
}

ent constituents (cellulose, hemicellulose and lignin), which have very different thermal behaviours. There is thus a need to develop laboratory tools that are able to predict the thermal and kinetic behaviour of biomasses from their composition.

Some authors have tried to predict thermal and kinetic behaviour of biomasses from their composition by using an additivity law. Rajeswara Rao and Sharma proposed a method in which the pyrolysis reaction is modelled by an $n$ th-order rate equation, for components of the biomass and also for the main biomass materials. They found that experimental data on different biomass materials can be satisfactorily predicted from the behaviour of their components and from their compositions assuming that there is no interaction between them [8].

Yang et al. [9] pyrolysed cellulose, hemicellulose and lignin, and synthesized biomass samples containing two or three of the biomass components. Samples were heated to $900^{\circ} \mathrm{C}$ at $10^{\circ} \mathrm{C} / \mathrm{min}$. The pyrolysis of the synthesized biomass samples indicated negligible interaction among the three components. A computational approach was made firstly to predict the weight loss of a synthesized biomass from its composition in cellulose, hemicellulose and lignin, and secondly to predict the proportions of the three components of a biomass. The results calculated for the weight loss of the synthesized biomass are quite consistent with the experimental results. However, results concerning the prediction of the composition of a biomass in cellulose, hemicellulose and lignin were not very satisfactory.

Biagini et al. studied TG-FTIR analysis of ligno-cellulosic biomasses. A constant heating rate of $20^{\circ} \mathrm{C} / \mathrm{min}$ was used in all the experimental runs, from 105 to $1000^{\circ} \mathrm{C}$. The aim of their 
work was to apply a weighted sum law (validated in the literature) for the TG results, and to validate and extend this law for the profiles of volatile species released and measured by FTIR. Assuming no interactions between the components, composition of biomass in cellulose, hemicellulose and lignin was deduced from the experiments. Once the chemical composition of the materials was obtained, a summative law was applied to the release of each gaseous species. The profiles of volatile species released were obtained with good agreement between the experimental results and the calculated values. However, experimental results are not in good agreement with the calculated values for paper sludge. This discrepancy can be ascribed to the predominant ash content of the material, which is expected to produce significant effects on the devolatilization reactions [10].

Caballero et al. studied the flash pyrolysis of almond shells and of their components in a Pyroprobe 1000 between 700 and $900{ }^{\circ} \mathrm{C}$. They found that the almond shell yields obtained with the Pyroprobe 1000 are close to those calculated from the yields from lignin, hemicellulose and cellulose for $\mathrm{CO}$ and $\mathrm{CO}_{2}$, but not always for light hydrocarbons. They studied the decomposition kinetics in a thermobalance too. The thermal global decomposition of almond shells cannot be reproduced by adding up the kinetics of decomposition of their fractions. The authors suggest that there are interactions between the components [11].

Miller and Bellan worked on the pyrolysis of biomass and of cellulose, hemicellulose and lignin. They developed a kinetic model to predict the pyrolysis of arbitrary biomass feedstocks - both in low temperature and in high temperature conditions - via a superposition of cellulose, hemicellulose and lignin kinetics. This model, which is based on the models of Bradbury et al. [12] and Di Blasi and Russo [13], is intended for typical feedstock specimens and atmospheric pyrolysis pressure. Further comparisons with the experiments that are not used to fit the kinetic parameters show a good agreement with the previous experiments of bagasse, cellulose, cherry wood, oak and pine for a large variety of both TGA and isothermal pyrolysis conditions. The kinetic schemes have even been incorporated into the previous porous particle model of Miller and Bellan in order to model macro-particle pyrolysis. However, this macro-particle model can be improved, and the authors quoted several possible explanations among which the mineral matter in the wood is one [14].

Finally, conclusions are different depending on the authors and on the experimental conditions. Some authors affirm that it is possible to use linear correlation to predict gas yields of a biomass from its composition, but others affirm that this is not possible and refer to possible interactions between the components and the likely effect of mineral matter.

Few studies have been done on the interactions between components, but we can find several works on the influence of mineral matter. Usually, two techniques are used to characterize the effect of mineral matter on pyrolysis reactions: biomass washing and biomass impregnation. Washing is generally done with water or with acid solutions. Depending on the technique, minerals are removed from the biomass to a greater or lesser degree. Extraction of minerals from biomass is always selective [15]. Moreover, washing biomass leads to a change in its composition in cellulose, hemicellulose, lignin and extractives. In fact, washing removes not only mineral matter but other components like extractives and a part of the hemicellulose too [17].

In the literature, results obtained after thermal treatment of washed biomass and impregnated biomass are in agreement [15-20]. The higher the concentration of minerals in biomass

- the higher the degradation rate;

- the lower the liquid yield;
- the higher the char yield;

- the higher the total gas yield. $\mathrm{H}_{2}$ and $\mathrm{CO}_{2}$ yields increase and $\mathrm{CH}_{4}$ and $\mathrm{CO}$ yields decrease.

The objective of this paper is to establish if it is possible, from an additivity law, to correlate the gas yields after flash pyrolysis at high temperature $\left(950^{\circ} \mathrm{C}\right)$ of any biomass from its composition in cellulose, hemicellulose and lignin.

The approach consisted in first pyrolysing real biomasses and searching for the gas yields of "theoretical components" (cellulose, hemicellulose and lignin) able to predict the gas yields of these biomasses after flash pyrolysis at high temperature. The first conclusion was that such a simple approach was not successful. The influence of interactions between the components and the effect of mineral matter on pyrolysis gaseous yields were then demonstrated and shown to be a possible cause of this conclusion.

\section{Experiments}

\subsection{Experimental device}

The entrained flow reactor (EFR) is described in Fig. 1. It is a $75 \mathrm{~mm}$ i.d. and $2 \mathrm{~m}$ long tube into which a $1 \mathrm{~m}$ long isothermal reaction zone is fed with an electrically preheated laminar gas flow. The powdered solid is injected through a water-cooled feeding probe and dispersed over the cross-section of the reactor using a dispersion dome. It is pyrolysed for a controlled residence time before being sampled by a water-cooled probe.

The solid injection consists of a low velocity conveyor belt that ensures an accurate solid mass flow rate. The belt is fed from a Vshaped rail along which a precisely weighted quantity of solid is regularly spread using a calibrated wedge. The gas and solid phases can be collected at different heights $(z)$ using a water-cooled sampling probe, allowing precise control of the residence time. After separating gas from particles, the gases are forwarded to the analysers, via a heated line

- a Fourier transform infrared (FTIR) analyser to quantify continuously $\mathrm{CO}, \mathrm{CO}_{2}, \mathrm{CH}_{4}, \mathrm{C}_{2} \mathrm{H}_{2}, \mathrm{C}_{2} \mathrm{H}_{4}, \mathrm{C}_{2} \mathrm{H}_{6}, \mathrm{C}_{3} \mathrm{H}_{8}, \mathrm{NO}, \mathrm{NO}_{2}, \mathrm{~N}_{2} \mathrm{O}$, $\mathrm{NH}_{3}, \mathrm{HCN}$ and $\mathrm{SO}_{2}$;

- a non-dispersive infrared (NDIR) analyser for $\mathrm{CO}, \mathrm{CO}_{2}, \mathrm{SO}_{2}$, $\mathrm{NO}$ and $\mathrm{NO}_{x}$ concentrations coupled with a paramagnetic analyser for $\mathrm{O}_{2}$ concentration;

- a thermal conductivity detector (TCD) for $\mathrm{H}_{2}$ concentration;

- a methane and total hydrocarbons analyser, using two flame ionisation detectors (FID);

During the experiments, $\mathrm{CO}, \mathrm{CO}_{2}, \mathrm{H}_{2}, \mathrm{CH}_{4}, \mathrm{C}_{2} \mathrm{H}_{2}$ and $\mathrm{C}_{2} \mathrm{H}_{4}$ were detected.

Gas sampling was operated at residence times up to $2.5 \mathrm{~s}$.

The atmosphere gas (nitrogen) and the reactor walls were heated to the controlled temperature of $950{ }^{\circ} \mathrm{C}$. The atmosphere gas plus the solid transport gas flow rate was $14 \mathrm{~L} / \mathrm{min}(12 \mathrm{~L} / \mathrm{min}$ for atmosphere gas and $2 \mathrm{~L} / \mathrm{min}$ for solid transport gas) at STP; the solid mass flow rate was $1 \mathrm{~g} / \mathrm{min}$.

\subsection{Properties of the solids used}

Because commercial hemicellulose cannot easily be purchased, xylan has been widely used as a representative of the hemicellulose component in pyrolysis processes.

For all products, the fraction between 50 and $125 \mu \mathrm{m}$ was selected by pneumatic sieving prior to any analysis and experiment. 


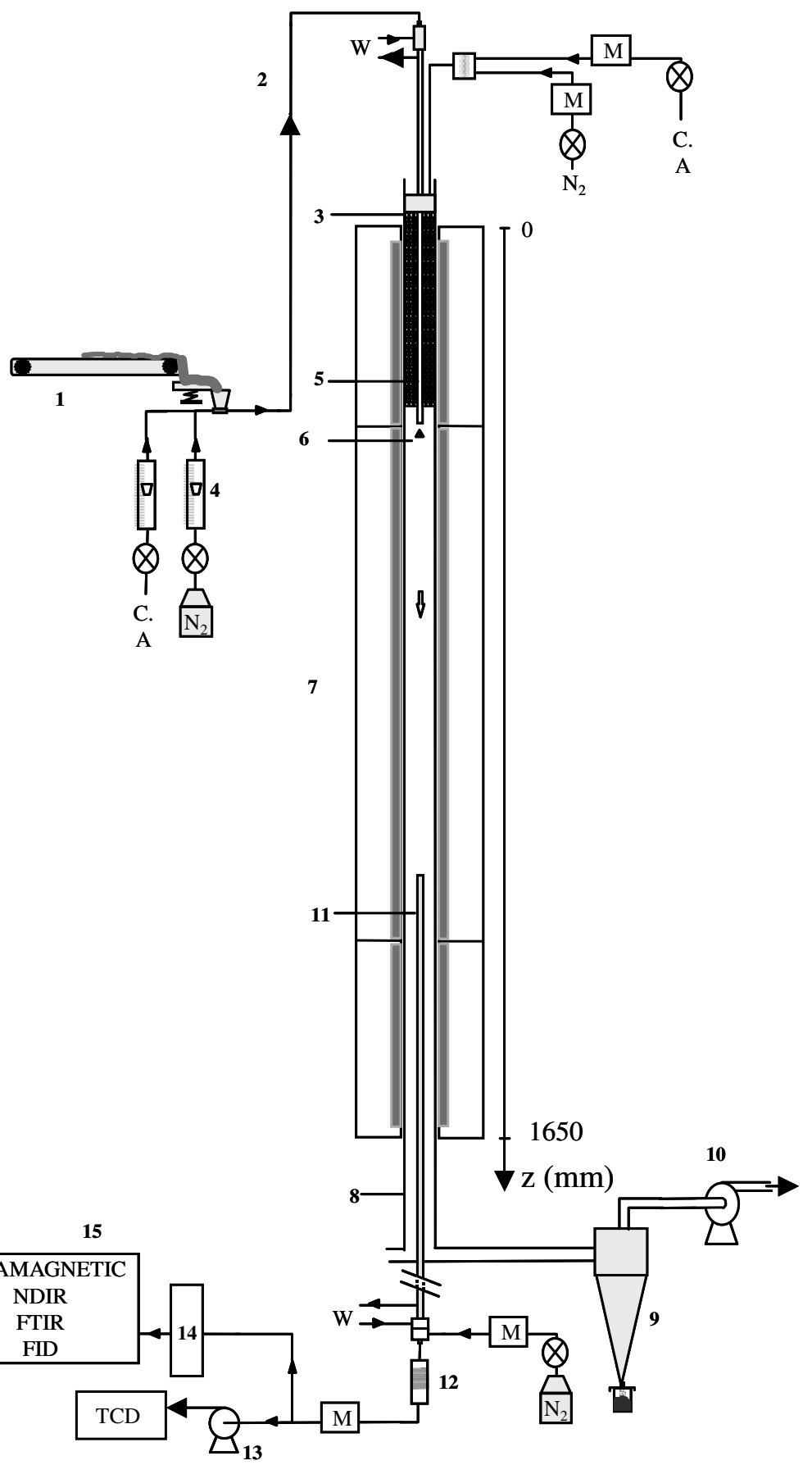

$\begin{array}{ll}\text { 1- Conveyor belt / vibrating corridor / ejector } & \text { 9- Cyclone collector } \\ \text { 2- Pneumatic transport } & \text { 10- Exhaust fan } \\ \text { 3- Electrical preheater } & \text { 11- Isokinetic water cooled } \\ \text { 4- Flow meters } & \text { sampling probe } \\ \text { 5- Water cooled feeding probe } & \text { 12- Particle collector (filter) } \\ \text { 6- Dispersion dome } & \text { 13- Sampling pump } \\ \text { 7- Three zones electrical furnace } & \text { 14- Gas conditioner } \\ \text { 8- 75mm i.d quartz tube reactor } & \text { 15- Gas analysers }\end{array}$

M- Mass flow meters and controllers $\mathrm{N}_{2}$ - Nitrogen

C.A- Compressed Air

W- Water (probes cooling)

12- Particle collector (filter)

Fig. 1. Schematic view of the entrained flow reactor of Ecole des Mines d'Albi.

The components used in this work were

- Two microcrystalline celluloses supplied by Sigma-Aldrich

- "microcrystalline cellulose"

- "Sigmacell microcrystalline cellulose"
- Three xylans supplied by Sigma-Aldrich

- xylan from birchwood

- xylan from beechwood

- xylan from oat spelts

- Three lignins 
- alkali lignin supplied by Sigma-Aldrich

- lignin supplied by Meadwestvaco

- lignin supplied by Borregaard

The extraction protocol that was used for each component is not known.

The biomasses used in this work were chosen because they have very different contents of cellulose, hemicellulose and lignin

- Beech wood I and beech wood II (supplied by Lignex)

- "Spruce and fir" mix (supplied by Lignex)

- rice husk (from Camargue, south-east of France)

- grass (from Albi, France)

- wood bark (from Les Landes, France)

As it is difficult to determine accurately the composition in cellulose, hemicellulose and lignin of a biomass because of the multitude of current extraction processes, average compositions found in the literature were adopted [21,22]. Grass contains large quantities of cellulose and hemicellulose and a very small amount of lignin. On contrary, wood bark contains a large quantity of lignin and very little cellulose. Wood (spruce and fir) contains the smallest quantity of hemicellulose.

Proximate analysis gave the amounts of ash, volatile matter (VM) and fixed carbon (complement to $100 \%$ ) as shown in Table 1. The amounts of carbon $(C)$, hydrogen $(H)$, nitrogen $(N)$ and sulphur $(\mathrm{S})$ were obtained by ultimate analysis. The amount of oxygen (O) was obtained as the complement to $100 \%$ of $\mathrm{C}, \mathrm{H}, \mathrm{N}, \mathrm{S}$ and ash.

Celluloses contain a large quantity of $\mathrm{O}$ and $\mathrm{H}$ in comparison with lignins. More unexpected are the differences observed inside a given family of components. For example, alkali lignin contains $37.1 \%$ of $\mathrm{O}$, while lignin from Meadwestvaco contains $26.1 \%$ of $\mathrm{O}$. Alkali lignin contains $56.6 \%$ of $\mathrm{C}$ and lignin from Meadwestvaco contains $66.7 \%$ of $\mathrm{C}$. As far as the biomasses are concerned, wood bark contains a large quantity of $\mathrm{C}$ but low quantities of $\mathrm{H}$ and $\mathrm{O}$ in comparison with the other biomasses.

Proximate analysis also indicates large differences. Celluloses contain ashes in undetectable quantities and very little fixed carbon; they form a large quantity of volatile matter during pyrolysis. Lignins contain large quantities of fixed carbon and produce little amounts of volatile matter.

Woody biomasses (beechwood and mix "spruce and fir") contain a small amount of ash in comparison with the other biomasses. They can form a large quantity of volatile matter during pyrolysis. Rice husk contains a large quantity of ash.

Mass fractions of cellulose, hemicellulose and lignin in each biomass are shown in Fig. 2.

\subsection{Preparation of the mixes}

As illustrated in Fig. 3, three-component mixes were performed and two ways

(i) Simple mix: The products were mixed in equal mass proportion with a spatula in a container. In this case, the particles, once injected in the EFR, may be in contact but will be essentially dispersed in the gas phase. In these conditions, interactions in gas phase are favoured.

(ii) Intimate mix: The components were mixed and then coground to thin elements (around $10 \mu \mathrm{m}$ ) using a laboratory ball mill. They were then agglomerated with a press, and then dispersed with a pestle and mortar to obtain particles around $100 \mu \mathrm{m}$. In this case, the thin elements of the components are in contact inside a given particle. Interactions between the components can now also occur inside the particles.

\subsection{Preparation of washed wood and impregnated wood}

\subsubsection{Washed wood}

Fifteen grams of beech wood II $(50-125 \mu \mathrm{m})$ was placed in $400 \mathrm{ml}$ of distilled water for $24 \mathrm{~h}$ to remove part of the mineral matter from the wood particles. After vacuum filtration, the "washed wood" was placed in a drying oven at $105{ }^{\circ} \mathrm{C}$ until evaporation of the water.

\subsubsection{Impregnated wood}

Firstly, straw ash was produced by burning straw in a muffle furnace at $815^{\circ} \mathrm{C}$ for $2 \mathrm{~h}$. Twenty-five grams of this ash was placed and shaken in $400 \mathrm{ml}$ of distilled water for $24 \mathrm{~h}$ to concentrate the water in the minerals. Secondly, $15 \mathrm{~g}$ of beech wood II was placed in the mineral concentrated water (after filtration) for $24 \mathrm{~h}$. Finally, after filtration, this "impregnated wood" was dried in an oven at $105^{\circ} \mathrm{C}$ until evaporation of the water.

Straw ash was chosen because straw contains a large quantity of minerals, especially $\mathrm{K}$ which is known to have a catalytic effect during thermal treatment of biomass [18].

Washing and impregnation of wood are known to affect the composition of wood by removing some extractives and hemicellulose. Consequently, "washed wood" and "impregnated wood" were prepared with the same mass of wood and of water. The aim was thus to affect the woods' compositions in cellulose, hemicellulose, lignin and extractives in the same manner. Mass loss caused by washing - calculated from weighing the dry wood before and after washing - represents $8.2 \%$ of the initial dry wood mass. Among this

Table 1

Ultimate analysis and proximate analysis of components and of biomasses

\begin{tabular}{|c|c|c|c|c|c|c|c|c|c|}
\hline & & \multicolumn{5}{|c|}{ Ultimate analysis (mass\% daf) } & \multicolumn{3}{|c|}{ Proximate analysis (dry mass\%) } \\
\hline & & $\mathrm{C}$ & $\mathrm{H}$ & $\mathrm{N}$ & $\mathrm{S}$ & 0 & Ash & Volatile matters & Fixed carbon \\
\hline \multirow[t]{8}{*}{ Components } & Microcrystalline cellulose & 44.4 & 5.8 & 0.3 & 0.1 & 49.3 & 0 & 94.8 & 5.2 \\
\hline & Sigmacell microcrystalline cellulose & 44.5 & 5.6 & 0.3 & 0.1 & 49.5 & 0 & 94.1 & 5.9 \\
\hline & Xylan from birchwood & 46.7 & 5.7 & 0.2 & 0 & 47.4 & 4.1 & 73.3 & 22.6 \\
\hline & Xylan from beechwood & 44.3 & 5.4 & 0.3 & 0 & 49.9 & 4.8 & 75.3 & 19.9 \\
\hline & Xylan from oat spelts & 44.8 & 5.6 & 0.04 & 0 & 49.5 & 10.1 & 71.1 & 18.8 \\
\hline & Alkali lignin & 56.6 & 4.2 & 0.5 & 1.6 & 37.1 & 10 & 49.9 & 40.1 \\
\hline & Lignin from Borregaard & 65 & 4.9 & 0.6 & 0.8 & 28.7 & 13.5 & 45 & 41.5 \\
\hline & Lignin from Meadwestvaco & 66.7 & 5.6 & 1.1 & 0.5 & 26.1 & 2.4 & 58.9 & 38.7 \\
\hline \multirow[t]{6}{*}{ Biomass } & Beechwood I & 46.1 & 5 & 0.03 & 0 & 48.9 & 0.5 & 84.3 & 15.2 \\
\hline & Beechwood II & 50.3 & 4.9 & 0 & 0 & 44.8 & 0.2 & 83.2 & 16.6 \\
\hline & Mix spruce and fir & 49.1 & 5.5 & 0 & 0 & 45.4 & 0.4 & 82.9 & 16.7 \\
\hline & Rice husk & 50.7 & 6.4 & 0.9 & 0.06 & 42 & 15.2 & 61.8 & 23 \\
\hline & Wood bark & 55.3 & 4.6 & 0.6 & 0 & 39.6 & 4.9 & 65.3 & 29.8 \\
\hline & Grass & 45.5 & 6 & 3.2 & 0 & 45.4 & 8.5 & 74.5 & 17 \\
\hline
\end{tabular}




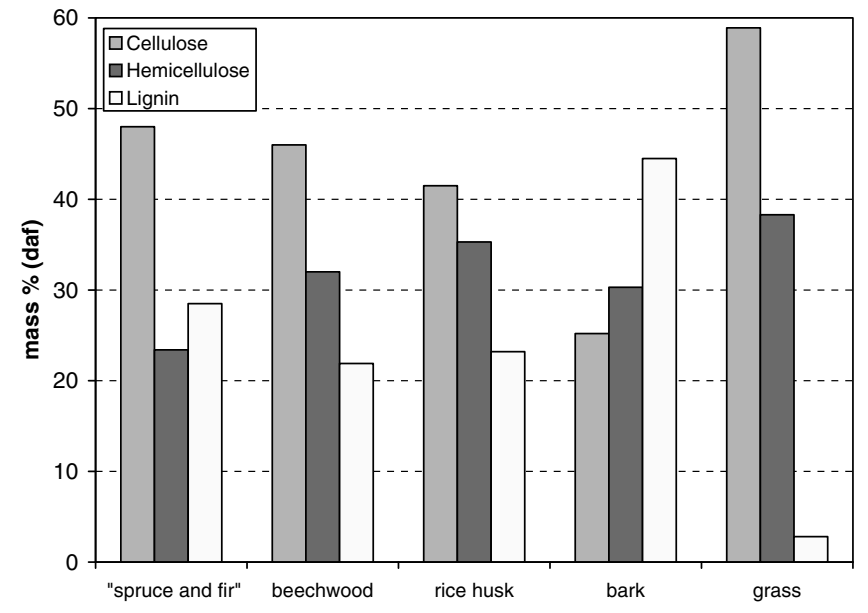

Fig. 2. Mass fractions of cellulose, hemicellulose and lignin in each biomass.

$8.2 \%$, only $0.02 \%$ represents mineral loss (calculated from proximate analysis). Finally, "washed wood" contained $0.2 \%$ of minerals (like the initial beech wood II) and "impregnated wood" contained $1.2 \%$ of minerals.

\section{Results and discussion}

3.1. Pyrolysis of biomasses and correlation between composition of the biomass and gas yields

\subsubsection{Pyrolysis of biomasses}

The beech wood I, "spruce and fir" mix, rice husk, grass and wood bark were pyrolysed in the EFR, and the resulting gases were analysed. Gas mass yields of biomasses were calculated (as at\% of the initial dry-ash-free (daf) biomass) and are shown in Fig. 4. Each experiment was repeated several times, typically 3-5 times; uncertainties were calculated as follows, and are indicated in the figure

unc $=\frac{\sum_{i=1}^{n}\left|X_{\mathrm{m}}-X_{i}\right|}{n}$

with $X_{\mathrm{m}}$, mean gas yield; $\mathrm{X}_{i}$, gas yield for experiment $i$; and $n$, number of experiments.

Note that the yield of $\mathrm{CO}$ and the total gas yield were divided by 10 in Fig. 4.

Gas yields are very different depending on the nature of the biomass. Results show that gas yields of the two woody biomasses (beech wood I and "spruce and fir" mix) are very similar. Moreover, they form more $\mathrm{CO}, \mathrm{CH}_{4}$ and $\mathrm{H}_{2}$ than the other biomasses. In fact, $\mathrm{CO}$ yield represents more than half of the total quantity of analysed gases. Consequently, a change in $\mathrm{CO}$ yield has a significant effect on the total quantities of analysed gases. This is why the total quantities of analysed gases are greater for the two woody biomasses than for all others biomasses.

Grass forms less $\mathrm{H}_{2}$ and $\mathrm{CO}$ than other biomasses; it forms a large quantity of $\mathrm{CO}_{2}$.

Note that after a time period of $0.5-1 \mathrm{~s}$, the gas yields of the biomasses do not appear to change significantly with time. Pyrolysis can be considered as finished after these short residence times. Moreover, it seems that gas phase cracking reactions stop forming permanent gas after these short residence times. It is not possible from the present results to determine accurately from what time the pyrolysis and tar cracking are over; this is not the scope of the work.

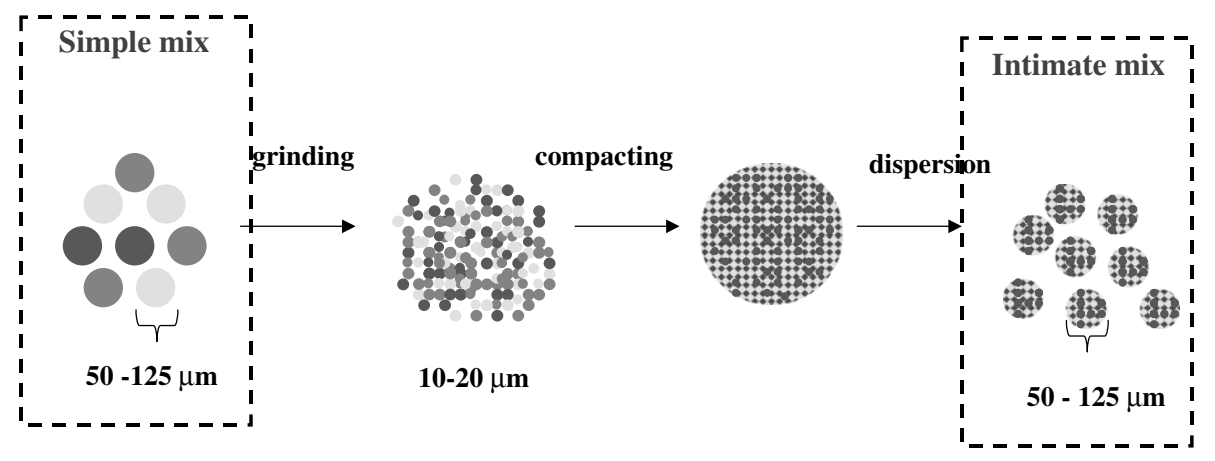

Fig. 3. Simple and intimate mixes.

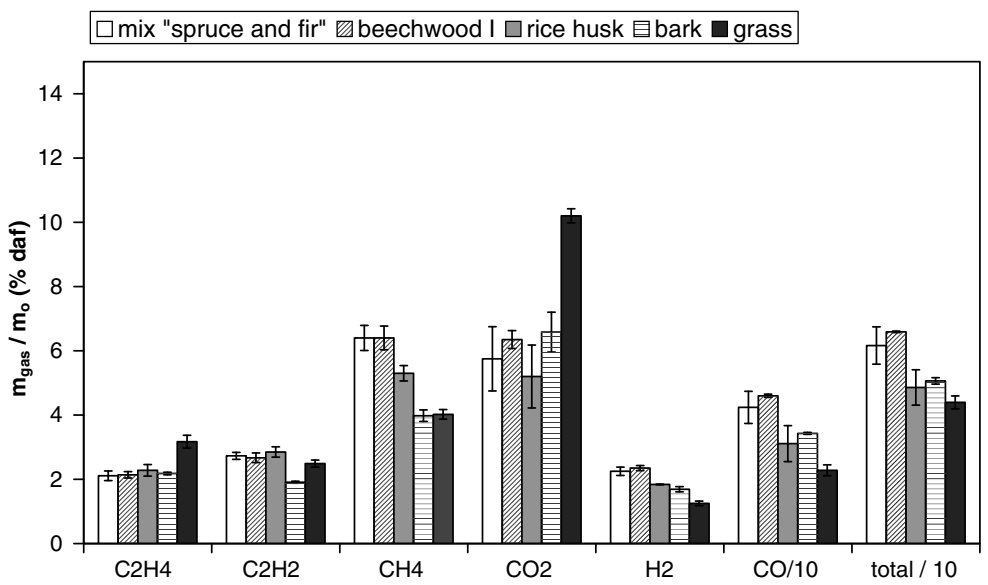

Fig. 4. Gas yields of biomasses. 


\subsubsection{Search for correlation}

In this study, we aim to research correlations between compositions of biomasses and gas yields obtained during their pyrolysis. Several approaches can be considered. Biomass can be described as a complex mix of polymers:

- composed of carbon, hydrogen and oxygen;

- composed of functional groups;

- composed of cellulose, hemicellulose and lignin.

In this work, we will focus on the search for correlation between the gas yields of any biomass and its composition in cellulose, hemicellulose and lignin.

If cellulose, hemicellulose and lignin in a biomass undergo pyrolysis without interaction between them, then gas yields should follow the additivity law
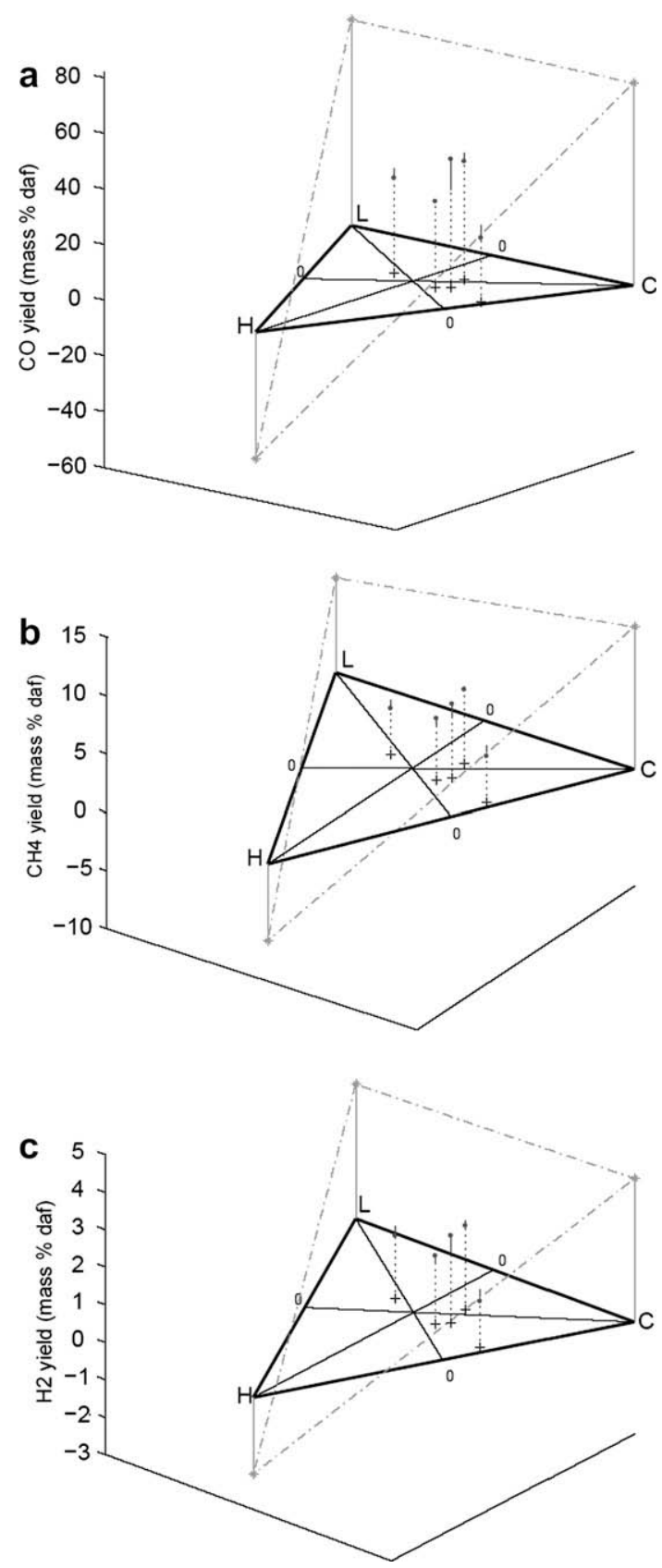

Fig. 5. Yields in $\mathrm{CO}(\mathrm{a}), \mathrm{CH}_{4}$ (b) and $\mathrm{H}_{2}$ (c) of biomasses and theoretical components.

$$
\begin{aligned}
& Y_{\text {beech wood }}=\alpha_{\text {beech wood }} Y_{\text {cellulose }}+\beta_{\text {beech wood }} Y_{\text {hemicellulose }} \\
& +\gamma_{\text {beech wood }} Y_{\text {lignin }} \\
& Y_{\text {spruce and fir }}=\alpha_{\text {spruce and fir }} Y_{\text {cellulose }}+\beta_{\text {spruce and fir }} Y_{\text {hemicellulose }} \\
& +\gamma_{\text {spruce and fir }} Y_{\text {lignin }} \\
& Y_{\text {rice husk }}=\alpha_{\text {rice husk }} Y_{\text {cellulose }}+\beta_{\text {rice husk }} Y_{\text {hemicellulose }}+\gamma_{\text {rice husk }} Y_{\text {lignin }} \\
& Y_{\text {bark }}=\alpha_{\text {bark }} Y_{\text {cellulose }}+\beta_{\text {bark }} Y_{\text {hemicellulose }}+\gamma_{\text {bark }} Y_{\text {lignin }} \\
& Y_{\text {grass }}=\alpha_{\text {grass }} Y_{\text {cellulose }}+\beta_{\text {grass }} Y_{\text {hemicellulose }}+\gamma_{\text {grass }} Y_{\text {lignin }}
\end{aligned}
$$

with $Y$, gas yield (for a given gas species); $\alpha_{i}$, mass fraction of cellulose in biomasses " $i$ "; $\beta_{i}$, mass fraction of hemicellulose in biomasses " $i$ "; $\gamma_{i}$, mass fraction of lignin in biomasses " $i$ ".

We have represented the experimental results of gas yields in Fig. 5 using a ternary diagram. This was done for the three main gases: $\mathrm{CO}, \mathrm{CH}_{4}$ and $\mathrm{H}_{2}$. The three vertexes are the pure components of cellulose, hemicellulose and lignin. The content of a biomass (+ symbols) in one component corresponds with the value read on the corresponding axis after perpendicular projection. In ordinate the gas yields are plotted.

If an additivity law can be applied, the gas yields for the five biomasses should lie in a plane. The intersection of this plane with the vertical lines above the vertexes $\mathrm{C}, \mathrm{H}$ and $\mathrm{L}$ would define the gas yields of "theoretical" pure components. We have searched this plane ("least squares plane") minimising standard deviations with
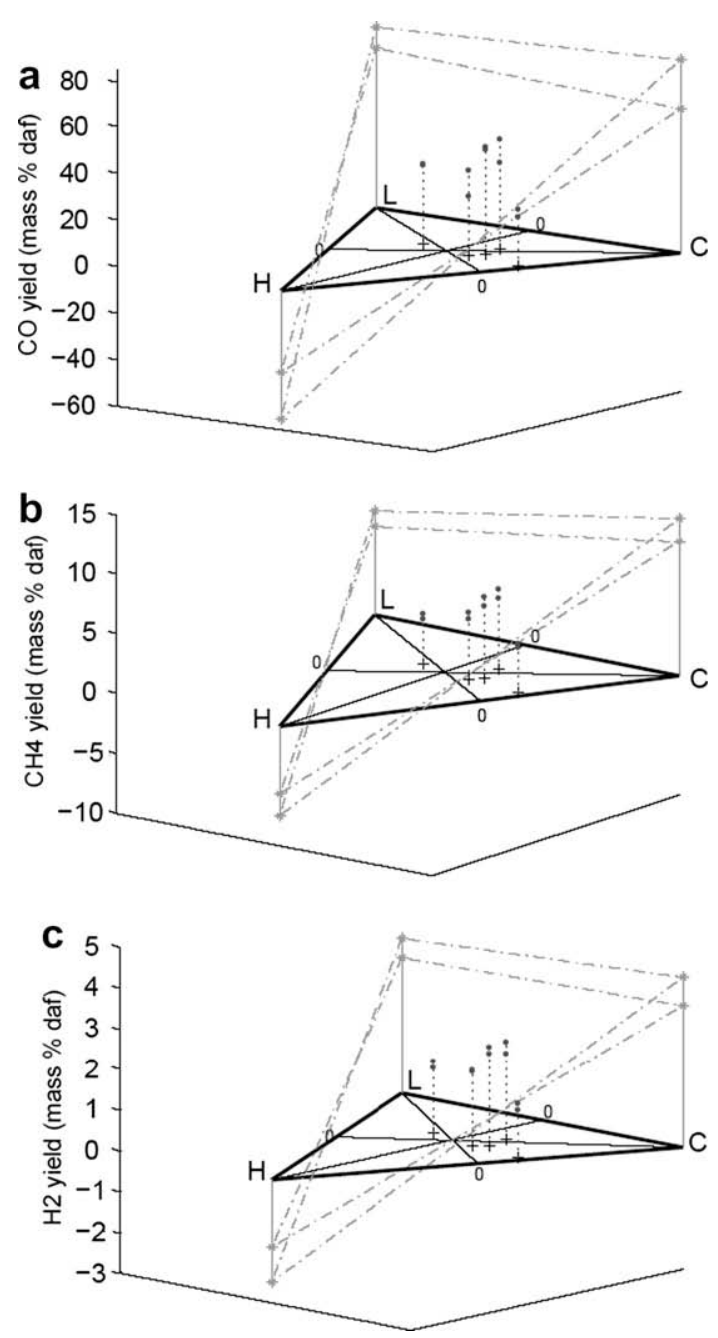

Fig. 6. Effect of uncertainties concerning yields in $\mathrm{CO}(\mathrm{a}), \mathrm{CH}_{4}$ (b) and $\mathrm{H}_{2}$ (c) of biomasses on yields of theoretical components. 
all the biomass gas yields. Discrepancies between the plane and the points corresponding to the biomass gas yields are materialised by continuous vertical lines. Fig. 5 shows that the intersection of the plane with the vertical line above the pure hemicellulose occurs at a negative value for $\mathrm{CO}, \mathrm{CH}_{4}$ and $\mathrm{H}_{2}$ yields, which is not a physically possible solution. At this stage, it would seem that we cannot find theoretical components able to represent the gas yields of the five biomasses.

However, it is necessary to take into account the uncertainties on gas yield values, because they can modify the position of the plane and thereby modify the gas yields of theoretical components. Therefore, two "least squares planes" are drawn in Fig. 6

- the least squares plane corresponding to the higher values of the biomass gas yields. These values are obtained by adding uncertainties to average values of gas yields (represented in Fig. 5).

- the least squares plane corresponding to the smallest values of the biomass gas yields. These values are obtained by subtracting uncertainties from average values of gas yields.

This shows that the intersections of the two planes with the vertical line above the pure hemicellulose still occur at negative values for the gas yield of hemicellulose. Consequently, even when uncertainties are taken into account, it is not possible to correlate gas yields of any biomass with its composition in cellulose, hemicellulose and lignin. This enables us to conclude that the idea that biomasses composed of the same cellulose, hemicellulose and lignin all pyrolyse independently, does not correspond to reality. It is also interesting to note that the least square planes always present a significant slope when varying only the hemicellulose content. This indicates that the amount of hemicellulose has a significant impact on all gas yields, while the impact of the ratio between lignin and cellulose seems less important.

A remark can be made concerning the possibility of using this ternary diagram representation for practical applications. We can see in Fig. 5 that the least squares plane is relatively close to the five points corresponding to the biomass gas yields in a limited area of biomass composition (located around the five biomasses). This plane can be used to predict gas yields of a biomass from its composition with a maximal discrepancy of $25 \%$, whereas gas yields of biomasses vary by $60 \%$. This result is interesting and needs to be confirmed with additional experiments of pyrolysis with alternative biomasses (with different compositions in cellulose, hemicellulose and lignin).
3.2. Failure of the component additivity rule to predict gas yields of biomass

It has been shown that it is not possible to predict the gas yields of any biomass from its composition in cellulose, hemicellulose and lignin. A number of potential explanations are quoted below:

- lignins in different biomasses may not be similar and may form different quantities of gases. The same explanation can be applied for hemicellulose and cellulose;

- components interact during pyrolysis;

- ash influences pyrolysis reactions;

- extractives contribute significantly to gas yields.

Hereafter, we will investigate the first three points in order to evaluate how far they can explain the discrepancies observed above.

\subsubsection{Gas yields of different lignins, hemicelluloses and celluloses}

Fig. 7 shows the values of gas yields of the different lignins, hemicelluloses and celluloses that were selected for this work, and characterized previously in detail. Again, the experiments were repeated 3-5 times, which makes it possible to indicate error bars on the figure.

It can be seen that there are differences between the average gas yields of the three different families of components. But these differences are often less significant than the differences observed inside a given family of components. For example, concerning $\mathrm{CH}_{4}$, the difference between yields of alkali lignin and lignin from Meadwestvaco is comparable to the difference between the average yield for lignins and the average yield for xylans. Such differences can be observed for the other gases yields too. However, the two celluloses produce similar quantities of gases. This is not surprising since the structure of cellulose is relatively well known and does not depend on its origin. It is not the case for lignins and hemicelluloses.

As was pointed out earlier, the differences between components' gas yields could perhaps be explained by the origin of the components, but may also be the result of the extraction process. Indeed, the structure of a component of the biomass can be modified by the extraction process, which may affect the gas yields during pyrolysis.

The conclusion on this point is that celluloses extracted from different biomasses lead to similar gas yields during pyrolysis. This

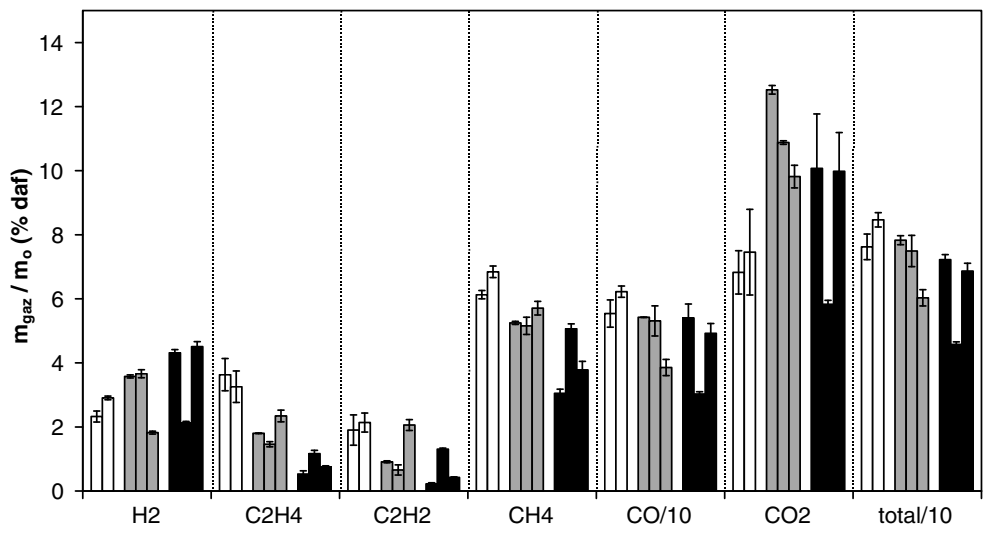

white: from left to right: microcrystalline cellulose and Sigmacell microcrystalline cellulose

grey : from left to right: xylan from beechwood, xylan from birchwood and xylan from oat spelts

black: from left to right: alkali lignin, lignin from Meadwestvaco and lignin from Borregaard.

Fig. 7. Gas yields from all the components at $950{ }^{\circ} \mathrm{C}$. 
is not the case for hemicellulose and for lignin, which can present very different yields in a same family. However, it cannot be established from this work whether differences are due to differences of original structures or if the extraction process has affected the structure of hemicellulose and lignin.

\subsubsection{Interactions between components}

In this study, we have carried out experiments to establish if interactions between components - inside or outside particles occur or not. For these experiments, microcrystalline cellulose, lignin from Borregaard and xylan from birch wood were first pyrolysed separately. They were then mixed in three-component mixes. The mixes were prepared in two ways: the simple mix and the intimate mix, as detailed before. The proportion of each component in the mix is similar to those found in natural birch wood: $40 \%$ cellulose, $39 \%$ hemicellulose and $21 \%$ lignin. If no interaction occurs, the gas yield (for a given gas species) of a mixture should be equal to

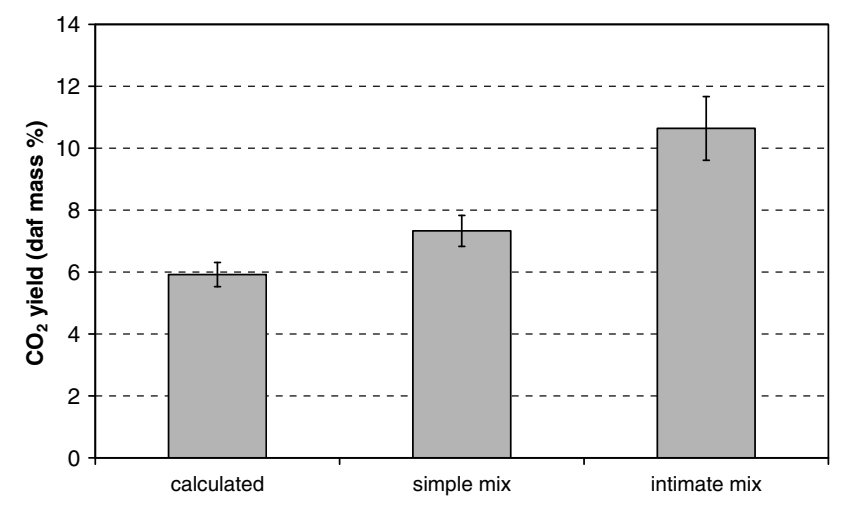

Fig. 8. $\mathrm{CO}_{2}$ yield calculated by Eq. (1) (considering no interaction) and $\mathrm{CO}_{2}$ yields measured during pyrolysis of simple and intimate mixes. the mass-weighted average yields of the three components as follows:

$Y_{\text {mix }}=0.4 Y_{\text {cel }}+0.39 Y_{\text {xyl }}+0.21 Y_{\text {lig }}$

where $Y_{\text {mix }}$ is the gas yield of the mix, $Y_{\text {cel }}$ is the gas yield of the cellulose, $Y_{\mathrm{xyl}}$ is the gas yield of the xylan, $Y_{\text {lig }}$ is the gas yield of the lignin.

The intimacy of the mixing might play a role on the interactions between the components. In order to take this into consideration, the differences between gas yields calculated with Eq. (1) and gas yields analysed during pyrolysis of simple and intimate mixings (described previously) have been plotted. Results - not presented here - have shown that it is difficult to conclude because of uncertainties (mean deviations obtained by repeating experiments three to five times) that are sometimes larger than the observed difference. However, for certain gas species, clear differences are observed between experimental yields and calculated yields. As an example, Fig. 8 presents the $\mathrm{CO}_{2}$ yields calculated with Eq. (1) and the $\mathrm{CO}_{2}$ yields measured during pyrolysis of simple and intimate mixes. Results show that there are differences between $\mathrm{CO}_{2}$ yield calculated with Eq. (1) and experimental $\mathrm{CO}_{2}$ yields. Moreover, there are significant differences in the $\mathrm{CO}_{2}$ yields of simple and intimate mixes: the more intimate the mix, the higher the $\mathrm{CO}_{2}$ yield.

A more detailed analysis of the situation is proposed before trying to interpret these results. During the pyrolysis of simple mixes, the three components devolatilize separately. Interactions are likely to occur outside the particles. Some potential interactions are described in Fig. 9 and quoted below:

1. Homogeneous gas phase reactions: gases and condensable vapours formed by one component may react with gases and condensable vapours formed by another component. Reforming, oxidation or polymerisation reactions are presumed.

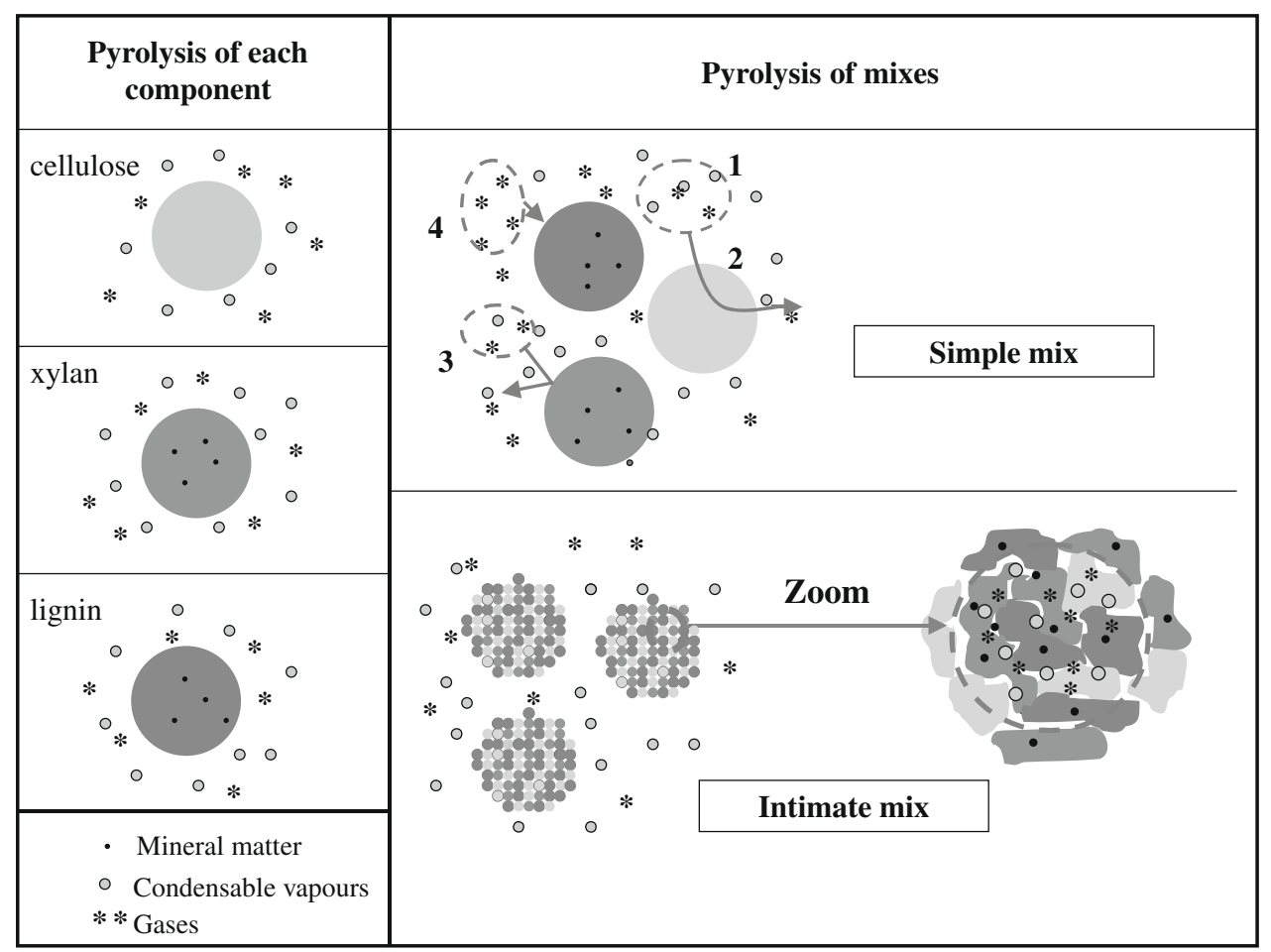

Fig. 9. Simplified schema of the pyrolysis of components taken separately and of the pyrolysis of component mixes. 


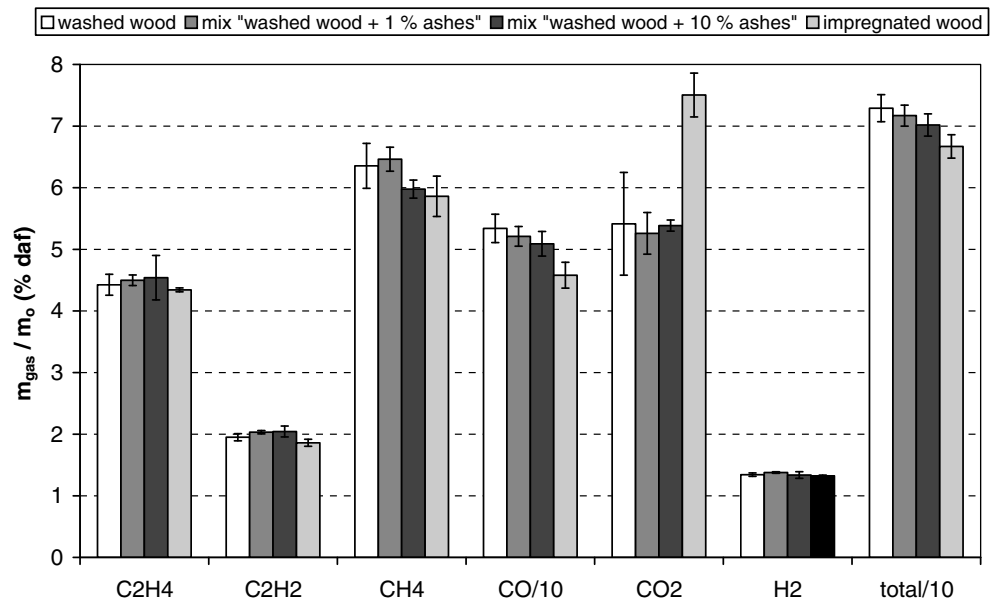

Fig. 10. Gas yields of "washed wood", "impregnated wood" and simple mixes "washed wood + ashes". Wood used is beech wood II.

2. Gas phase reactions: catalysed by char or/and ashes (or mineral matter), like for example catalysed cracking and polymerisation reactions. Char could modify $\mathrm{CO}-\mathrm{CO}_{2}$ equilibrium too.

3. Heterogeneous reactions: gases formed by one component may react with char formed by another component. For example, char oxidation reactions by $\mathrm{O}_{2}, \mathrm{H}_{2} \mathrm{O}$ or $\mathrm{CO}_{2}$ may occur. These reactions could be catalysed by ashes or mineral matter.

4. Influence of pyrolysis atmosphere: gases formed by one component may influence the devolatilization process of another component by modifying the atmosphere under which it pyrolyses.

During the pyrolysis of intimate mixes, reactions can occur outside the particles in the same way as during the pyrolysis of simple mixes, but additional interactions may occur inside the particles. As one component devolatilizes inside the particle, it is submitted to an atmosphere with very high concentrations in gas and condensable vapours; the gases formed are in close contact with the solids of other components.

In the present results, there are interactions during the pyrolysis of simple mix. Consequently, it is likely that some interactions occur outside the particles because particles are not in contact during this pyrolysis. There are also probably interactions inside the particles because $\mathrm{CO}_{2}$ yield of intimate mix is higher than $\mathrm{CO}_{2}$ yield of simple mix. Similar results were obtained in a previous work [23] with two component mixes instead of three-component mixes in this work.

\subsubsection{Influence of mineral matter}

In this part, the aim is to evaluate the potential influence of mineral matter on gas yields in the conditions of high temperature rapid pyrolysis. An attempt is made to differentiate the effect of minerals located inside a particle and the effect of minerals when dispersed in the gas phase.

A "washed wood" containing $0.2 \%$ of minerals and an "impregnated wood" containing $1.2 \%$ of minerals were prepared as detailed previously to study the effect of minerals inside the particle. In parallel, simple mixes "washed wood $+1 \%$ straw ash" and "washed wood $+10 \%$ straw ash" were prepared by manual mixing in order to investigate the second effect.

Fig. 10 shows gas yields of "washed wood", "impregnated wood" and the two simple mixes.

"Washed wood", the simple mix "washed wood + 1\% straw ash" and the simple mix "washed wood $+10 \%$ straw ash" form approximately the same quantities of gases even if a slight decrease in the yields of $\mathrm{CH}_{4}$ and $\mathrm{CO}$ is observed when the amount of ash is increased in the phase gas. It can be concluded that adding ash in the gas phase in quantities of $6.10^{-4} \mathrm{~g} / \mathrm{g}$ gas and $6.10^{-3} \mathrm{~g} / \mathrm{g}$ gas only slightly affects the observed gas yields after pyrolysis.

On the other hand, "impregnated wood" forms less $\mathrm{CO}$, much more $\mathrm{CO}_{2}$ and less $\mathrm{CH}_{4}$ than "washed wood". The $\mathrm{H}_{2}$ yield is not affected by ash enrichment. We can conclude that minerals significantly influence the pyrolysis reactions occurring inside the particle.

\section{Conclusions and perspectives}

The main result from this study is that it is not possible to predict - from an additivity law - pyrolysis gas yields of any biomass from its composition in cellulose, hemicellulose and lignin. This work shows that two phenomena may explain this:

- components interact between themselves during pyrolysis. It was shown in this work that interactions occur outside the particle - i.e. in the gas phase - and probably also inside the particles since the intimacy of the mix has an impact on the $\mathrm{CO}_{2}$ yields and

- minerals influence pyrolysis reactions occurring inside the particle. They favour the formation of $\mathrm{CO}_{2}$ and decrease the formation of $\mathrm{CO}$ and $\mathrm{CH}_{4}$; an addition up to $6.10^{-3} \mathrm{~g} / \mathrm{g}$ gas of ash in gas phase does not influence the reactions occurring outside the particle.

The approach consisting of extracting pure components from biomass in order to characterize their behaviour during pyrolysis is not recommended. Indeed, it remains impossible to establish how far the extraction process affects the structure of components.

This research work also yields practical information. It seems possible - in a restricted domain of composition - to predict the gas yields of any biomass from its composition in cellulose, hemicellulose and lignin, with a maximal discrepancy of $25 \%$, whereas gas yields of biomasses vary by $60 \%$. This result needs to be confirmed with additional experiments of pyrolysis of alternative biomasses.

\section{Acknowledgements}

We thank the GEM (Groupe des Ecoles des Mines) for supporting the " $\mathrm{H}_{2}$-fuel cell" project and CNRS for supporting the "Biomasters" Project.

\section{References}

[1] Ferdous D, Dalai AK, et al. Production of $\mathrm{H}_{2}$ and medium Btu gas via pyrolysis of lignins in a fixed-bed reactor. Fuel Processing Technol 2001;70:9-26. 
[2] Demirbas A. Gaseous products from biomass by pyrolysis and gasification: effects of catalyst on hydrogen yield. Energy Convers Manage 2002;43: 897-909.

[3] Corella J, Monzon A, et al. Ultra-fast biomass pyrolysis in a high-temperature $\left(2200^{\circ} \mathrm{C}\right)$, fluid-wall reactor. J Solar Energy Eng 1988;110:10-3.

[4] Zanzi R, Sjöström K, et al. Rapid pyrolysis of agricultural residues at high temperature. Biomass Bioenergy 2002;23:357-66.

[5] Li S, Xu S, et al. Fast pyrolysis of biomass in free-fall reactor for hydrogen-rich gas. Fuel Processing Technol 2004;85:1201-11.

[6] Bitowft B, Andersson LA, et al. Fast pyrolysis of sawdust in an entrained flow reactor. Fuel 1989;68:561-6.

[7] Zanzi R, Sjöström K, et al. Rapid high-temperature pyrolysis of biomass in a free-fall reactor. Fuel 1996;75(5):545-50.

[8] Rajeswara Rao T, Sharma A. Pyrolysis rates of biomass materials. Energy 1998;23(11):973-8.

[9] Yang $\mathrm{H}$, Yan R, et al. In-depth investigation of biomass pyrolysis based on three major components: hemicellulose, cellulose and lignin. Energy Fuels 2006;20:388-93.

[10] Biagini E, Barontini F, et al. Devolatilization of biomass fuels and biomass components studied by TG/FTIR technique. Ind Eng Chem Res 2006;45: 4486-93.

[11] Caballero JA, Font R, et al. Comparative study of the pyrolysis of almond shells and their fractions, holocellulose and lignin Product yields and kinetics. Thermochim Acta 1996;276:57-77.

[12] Bradbury AG, Sakai Y, Shafizadeh F. A kinetic model for pyrolysis of cellulose. J App Polym Sci 1979;23:3271-80.
[13] Di Blasi C, Russo G. Modeling of transport phenomena and kinetics of biomass pyrolysis. In: Bridgwater AV, editor. Advances in thermochemical biomass conversion, vol. 2. New York: Blackie Academic and Professional; 1994. p. 906-21.

[14] Miller RS, Bellan J. A generalized biomass pyrolysis model based on superimposed cellulose, hemicellulose and lignin kinetics. Combust Sci Technol 1997;126:97-137.

[15] Di Blasi C, Branca C, et al. Degradation characteristics of straw and washed straw. Thermochim Acta 2000;364:133-42.

[16] Das P, Ganesh A, et al. Influence of pretreatment for deashing of sugarcane bagasse on pyrolysis products. Biomass Bioenergy 2004;27:445-57.

[17] Yang H, Yan R, et al. Influence of mineral matter on pyrolysis of palm oil wastes. Combustion Flame 2006;146:605-11.

[18] Nik-Azar M, Hajaligol MR, et al. Mineral matter effects in rapid pyrolysis of beech wood. Fuel Processing Technol 1997;51:7-17.

[19] Bru K, Blin J, et al. Pyrolysis of metal impregnated biomass: an innovative catalytic way to produce gas fuel. J Anal Appl Pyrolysis 2007;78:291-300.

[20] Vamvuka D, Troulinos S, et al. The effect of mineral matter on the physical and chemical activation of low rank coal and biomass materials. Fuel 2006;85: 1763-71.

[21] Energy Research Centre of the Netherlands. <http://www.ecn.nl/phyllis/single. html>; 2006.

[22] Williams PT, Nugranad N. Comparison of products from the pyrolysis and catalytic pyrolysis of rice husks. Energy 2000;25:493-513.

[23] Couhert C, Commandré JM, Salvador Sylvain. Failure of the component additivity rule to predict gas yields of biomass in flash pyrolysis at $950{ }^{\circ} \mathrm{C}$. Biomass Bioenergy, in press. doi: 10.1016/j.biombioe.2008.07.003. 